\title{
Configural and contextual prioritization in object-based attention
}

\author{
SARAH SHOMSTEIN and STEVEN YANTIS \\ Johns Hopkins University, Baltimore, Maryland
}

\begin{abstract}
When attention is directed to a location within an object, other locations within that object also enjoy an attentional advantage. Recently we demonstrated that this object-based advantage is mediated by increased attentional priority assigned to locations within an already attended object and not to early sensory enhancement due to the "spread" of attention within the attended object (Shomstein \& Yantis, 2002). At least two factors might contribute to the assignment of attentional priority, one related to the configuration of objects in a scene and the other related to the probability of target appearance in each location imposed by task contingencies. We investigated the relative contribution of these factors by cuing one end of one of a pair of rectangles; a subsequent target appeared most often in the cued location. We manipulated attentional priority setting by varying (1) the probability that a target would appear in each of two uncued locations and (2) the cue to target stimulus onset asynchrony (SOA). On invalidly cued trials, the target appeared in the high-probability location (defined by an absolute spatial location, e.g., upper right) $83 \%$ of the time and in the low-probability location (e.g., lower left) $17 \%$ of the time. In both conditions, uncued targets appeared in the cued object half the time and in the uncued object half the time. At short SOAs, the same-object and probability effects were approximately additive. However, at longer SOAs, the same-object effects disappeared, and reaction times depended exclusively on location probability. These results suggest that observers adopt an implicit configural scanning strategy (in which unattended locations within an attended object have high priority) or an implicit contextual scanning strategy (in which objectively high-probability locations have high priority) depending on task contingencies and the amount of time that is available to deploy attention.
\end{abstract}

Studies of object-based visual attention have focused on how object boundaries and object parts affect the deployment of attention in a scene. Most of the early research on this topic sought to demonstrate the existence of object-based selection and to compare it with spacebased selection (e.g., Behrmann, Zemel, \& Mozer, 1998; Egly, Driver, \& Rafal, 1994; Moore, Yantis, \& Vaughan, 1998; Rock \& Gutman, 1981; Watson \& Kramer, 1999; for review, see Egeth \& Yantis, 1997). Recent work has probed the mechanisms of object-based attentional selection (Avrahami, 1999; Cepeda \& Kramer, 1999; Shomstein \& Yantis, 2002).

We recently postulated two possible accounts for objectbased attention (Shomstein \& Yantis, 2002). According to the early sensory enhancement account, following the biased competition model of Desimone and Duncan (1995),

Portions of this work were presented at the 2002 Vision Sciences meeting in Sarasota, Florida. Support was provided by NIH Grants R01-MH43924 and R01-DA13165 to S.Y. We thank Art Kramer, Zhe Chen, Cathleen Moore, and an anonymous reviewer for helpful suggestions. Correspondence should be addressed to S. Shomstein, who is now at the Department of Psychology, Carnegie Mellon University, Pittsburgh, PA 15213 (e-mail: shomstein@cmu.edu). cortical object representations are mutually inhibitory, so that the strength of a representation is weaker when an object is presented in a multielement scene than when presented alone (e.g., Reynolds, Chelazzi, \& Desimone, 1999). Following a cue or an instruction, a top-down biasing signal strengthens the sensory representation of the attended location or object and causes it to more effectively compete with other representations. According to this account, the deployment of attention to part of an object strengthens the representation of the entire object, an effect that may be likened to the "spread of attention" throughout the object.

Alternatively, an attentional prioritization process may affect the order in which different regions of the scene are visually investigated. According to this account, there is an inherent predisposition to assign higher attentional priority to locations within an already attended object than to locations elsewhere. Because attentional priority determines the order in which different regions of the scene are investigated, unattended parts of an attended object will enjoy an attentional advantage over other objects and locations in a scene.

Both of these accounts could explain the pattern of results from paradigms that require attention to be de- 
ployed to multiple parts of the scene over time (e.g., Behrmann et al., 1998; Egly et al., 1994; Moore et al., 1998; Watson \& Kramer, 1999). However, the two accounts make different predictions under some circumstances. Shomstein and Yantis (2002) asked subjects to identify a central target while attempting to ignore flanking distractors that were mapped to either a compatible or an incompatible response (following the classic flankers paradigm of Eriksen and colleagues; e.g., Eriksen \& Eriksen, 1974). The flanking letters appeared either within the object occupied by the target or at an equidistant location in a different object. An important feature of this design was that spatial location of the target was known with $100 \%$ certainty. According to the early sensory enhancement account, attention should "spread" within the attended object so that the flanking letters located in that object should benefit perceptually relative to objects elsewhere. This account predicts, therefore, that the time to identify the target letter will depend on the identity (or more precisely on the response compatibility) of the flanking letters to a greater degree when they appear in the attended object than when they appear in the unattended object. In contrast, the attentional prioritization account predicts that because the target location is known with $100 \%$ certainty, only one location should be examined in order to perform the task, so the priority of other locations in the scene remains near zero. Therefore, this account predicts that flanker interference will not depend on whether the flankers occupy the same or different object as the target (although the absolute spatial separation between the target and flankers may well affect performance due to spatial "leakage" of attention). The predictions of the prioritization account, and not of the early sensory enhancement account, were satisfied.

A critical further test of the predictions of the two accounts was to reduce target location certainty from $100 \%$ to equiprobable among four locations. In this case, multiple locations are potentially relevant, and any objectbased prioritization bias should be evident. Under these conditions, object-based modulation of the flanker effect was observed. These results suggest that object-based selection may reflect an implicit object-specific attentional prioritization strategy that arises only when multiple locations in the scene must be attended. We shall refer to this object-based prioritization strategy as configuralbecause it is triggered by the configuration of features in the scene following long-term perceptual learning that emphasizes the importance of objects. Attentional priority may also depend on the current behavioral context (e.g., cue validity); we shall refer to such prioritization strategies as context dependent.

Most previous results can be understood in these terms. For example, subjects in Experiment 1 of Egly et al. (1994) assigned attentional priority to locations in the scene according to both context-dependent and configural cues. The task context required that the highest attentional priority be assigned to the cued location, which was most likely to contain the target, intermediate prior- ity to the two uncued but potential target locations, and low priority everywhere else in the scene (where targets could never appear). The configural object-based prioritization bias would lead to the assignment of relatively high priority to all parts of the cued object. The combination of these two factors would yield a priority map in which the cued location has the highest priority, the uncued location in the attended object has the next highest priority, the uncued target location in the unattended object has lower priority, and the rest of the scene has very low priority. This matches the pattern of results reported in that study.

The purpose of the present study was to dissociate these two effects. We reasoned that over time the two factors may compete for control over priority assignment, and so we varied the cue-target stimulus onset asynchrony (SOA) to determine whether the relative contributions of the two sources of priority differ over time.

\section{EXPERIMENT 1}

Experiment 1 provided a manipulation check for the paradigm to be used in Experiment 2. The experiment was designed to confirm that observers can implicitly learn the probabilities with which targets appear in display locations during a cued attention task and use this contextual knowledge to deploy attention in the scene.

\section{Method}

Subjects. Fifteen Johns Hopkins University undergraduates participated for extra credit in a psychology course. All reported normal or corrected-to-normal visual acuity.

Apparatus and Stimuli. Stimuli were displayed on a 19-in. color monitor. A chinrest maintained a fixed viewing distance of $62 \mathrm{~cm}$. A central $0.3^{\circ} \times 0.3^{\circ}$ fixation square and four white square outlines appeared on a black background. Each square subtended $1.2^{\circ} \times 1.2^{\circ}$ of visual angle with $1.8^{\circ}$ separation from the neighboring squares (edge to edge).

One target ( $\mathrm{T}$ or $\mathrm{L})$ and three distractors (T/L hybrid) appeared on each trial; each item was centered within a square. Target letters were constructed with line segments each subtending $0.6^{\circ}$ of visual angle. Distractor items were created by superimposing the two target letters (i.e., $\mathrm{T}$ and $\mathrm{L}$ ). Target and distractor letters were presented in one of four possible orientations - upright, or rotated $90^{\circ}$, $180^{\circ}$, or $270^{\circ}$ (Figure 1). The cue consisted of the appearance of a red square that perfectly circumscribed one of the white squares (no gap) for $100 \mathrm{msec}$. Both target and distractor letters were presented in blue and subtended $0.6^{\circ} \times 0.6^{\circ}$ of visual angle.

Design and Procedure. A nested experimental design with three levels of the cuing factor was employed: valid cue, invalid cue/high probability, and invalid cue/low probability. Only the upper left or lower right square was cued (each on $50 \%$ of the trials). The target appeared in the cued location on half the trials. On $41.7 \%$ of the trials (i.e., $83 \%$ of the invalidly cued trials), the target appeared in the high-probability square and on $8.3 \%$ of the trials (i.e., on $17 \%$ of the invalidly cued trials), the target appeared in the low-probability square. For half the subjects, the high-probability square was located in the upper right and the low-probability square was in the lower left, whereas for the other half, these positions were reversed. The absolute locations of the cue and the high- and lowprobability squares were not expected to exert a systematic influence.

Each trial began with a display that consisted of a fixation cross and four squares presented for $1,000 \mathrm{msec}$. The cue was then flashed for $100 \mathrm{msec}$ after an additional $100 \mathrm{msec}$ by the target and 


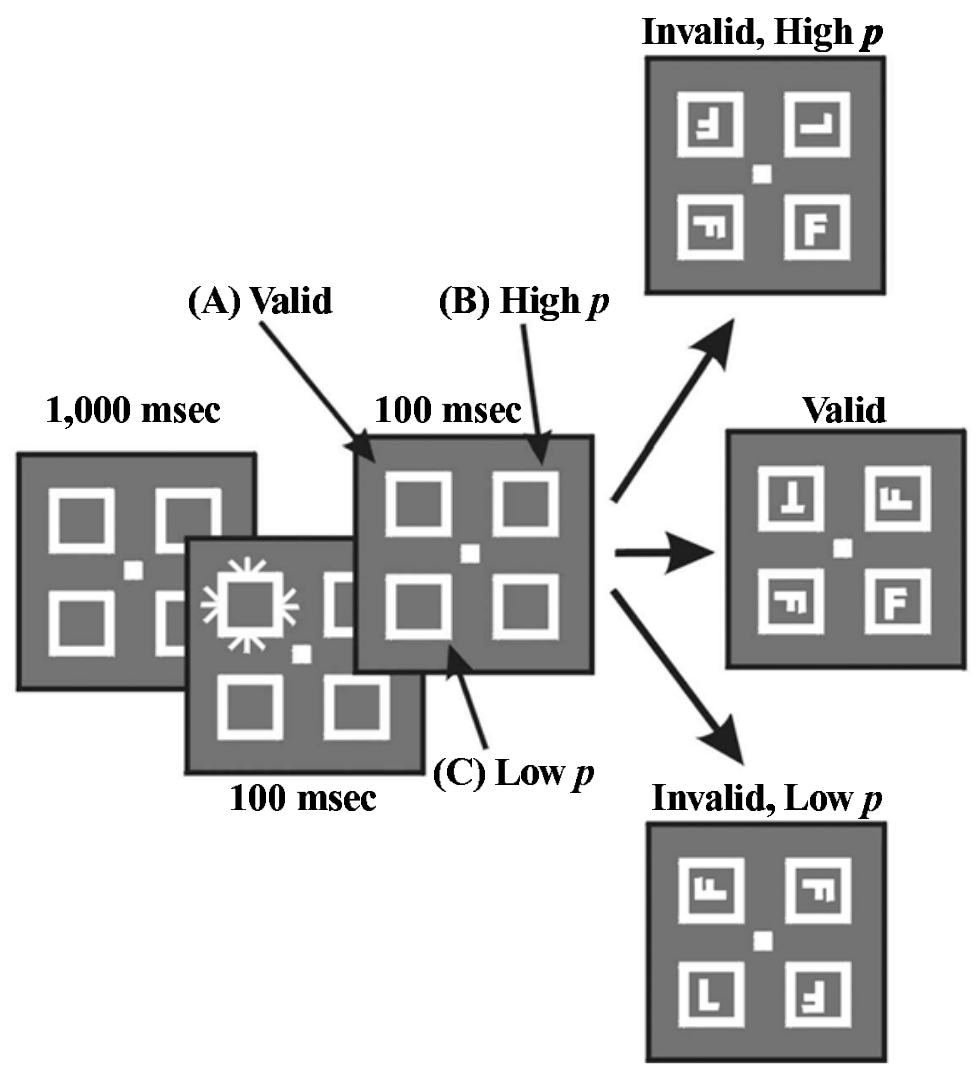

Figure 1. Display for each of the three conditions of Experiment 1. The task was to perform a $\mathrm{T} / \mathrm{L}$ letter discrimination among $\mathrm{T} / \mathrm{L}$ hybrid nontargets. (A) Valid target location: The target appears in the cued square. (B) Invalid high-probability location: upper right. (C) Invalid low-probability location: lower left (this mapping was reversed for half of the subjects).

three distractors, which remained until the subject responded. The subjects' task was to identify the target as either T or L (each occurred on a randomly selected half of the trials). Subjects were instructed to ignore the rotation and report only the identity of the target letter, and to respond as quickly as possible while remaining fixated and maintaining about $95 \%$ accuracy. The intertrial interval (ITI) was 2,000 msec following each correct response. Each error was followed by a beep and an ITI of 4,000 msec. Upon the completion of each block of trials, participants were presented with feedback for that block including their mean reaction time (RT) and error rate. Each subject participated in two 1-h sessions each consisting of nine blocks of 96 trials, for a total of 864 trials per session.

\section{Results}

RTs for correct responses only were analyzed. Because subjects were not told about the probability manipulation, the first experimental session was treated as a learning session and those data are not reported here. RTs greater than $1,500 \mathrm{msec}$ were removed from the analysis, resulting in the discarding of less than $1 \%$ of trials. Preliminary analysis showed that there were no significant main effects or interactions involving the identity of the target (T vs. L), the position of the cue (upper left vs. lower right), or the position of the high- and low-probability squares (all $F \mathrm{~s}<1$ ), and therefore the data were collapsed across the levels of these factors.

A one-way repeated measures analysis of variance (ANOVA) was conducted with cue validity (valid, invalid high-probability, invalid low-probability) as a withinsubjects factor and RT as the dependent measure. The ANOVA revealed a significant main effect of validity $[F(2,28)=32.12, p<.001]$ : RTs for valid targets $(M=$ $689 \mathrm{msec}$ ) were faster than those for either invalid highprobability $(M=718 \mathrm{msec})$ or invalid low-probability $(M=782 \mathrm{msec})$ targets. A planned comparison revealed that invalid low-probability targets were identified significantly more slowly than invalid high-probability targets $[F(1,14)=23.97, p<.001]$. In addition, a planned comparison revealed a significant difference between the valid and the invalid high-probability conditions $[F(1,14)=$ $12.75, p<.05]$; this shows that the effect was not due solely to very slow responses in the invalid low-probability condition.

In addition, a one-way repeated measures ANOVA was conducted with cue validity as the within-subjects factor and error rate as the dependent measure. The ANOVA revealed a significant main effect of validity $[F(2,28)=$ 
$4.05, p<.05]$. In addition, a planned comparison revealed a marginally significant difference between the invalid high-probability and the invalid low-probability conditions $[F(1,14)=4.35, p=.05]$. The percent error rate was $2 \%, 2 \%$, and $3 \%$ for valid, high-probability, and low-probability conditions, respectively. There is no indication of a speed-accuracy tradeoff-responses in the low-probability condition were both slower and more error prone than those in the high-probability condition.

\section{Discussion}

The results of Experiment 1 show that subjects can learn a simple probability distribution that is imposed on potential target locations and use this (intentionally or not) to deploy attention in the scene. The magnitude of the probability manipulation $(64 \mathrm{msec})$ will serve as a reference for the experiment reported below.

This result corroborates earlier studies conducted over the past 30 years showing that target location probabilities can influence the way in which a visual display is searched (e.g., Geng \& Behrmann, 2002; Miller, 1987; Moore \& Egeth, 1998; Shaw, 1978). The present experiment confirms that these findings apply to the present paradigm.

\section{EXPERIMENT 2}

So far we have demonstrated that the attentional prioritization map can be influenced by manipulating the probability of target locations. In Experiment 2, we examined the relative contribution of context-dependent (i.e., probability-based)and configural (i.e., object-based) priorities on performance.

\section{Method}

Subjects. Three groups of 18 new subjects in each group from the Johns Hopkins University community participated in return for extra credit in a psychology course. All subjects reported normal or corrected-to-normal visual acuity and were naive as to the purpose of the experiment.
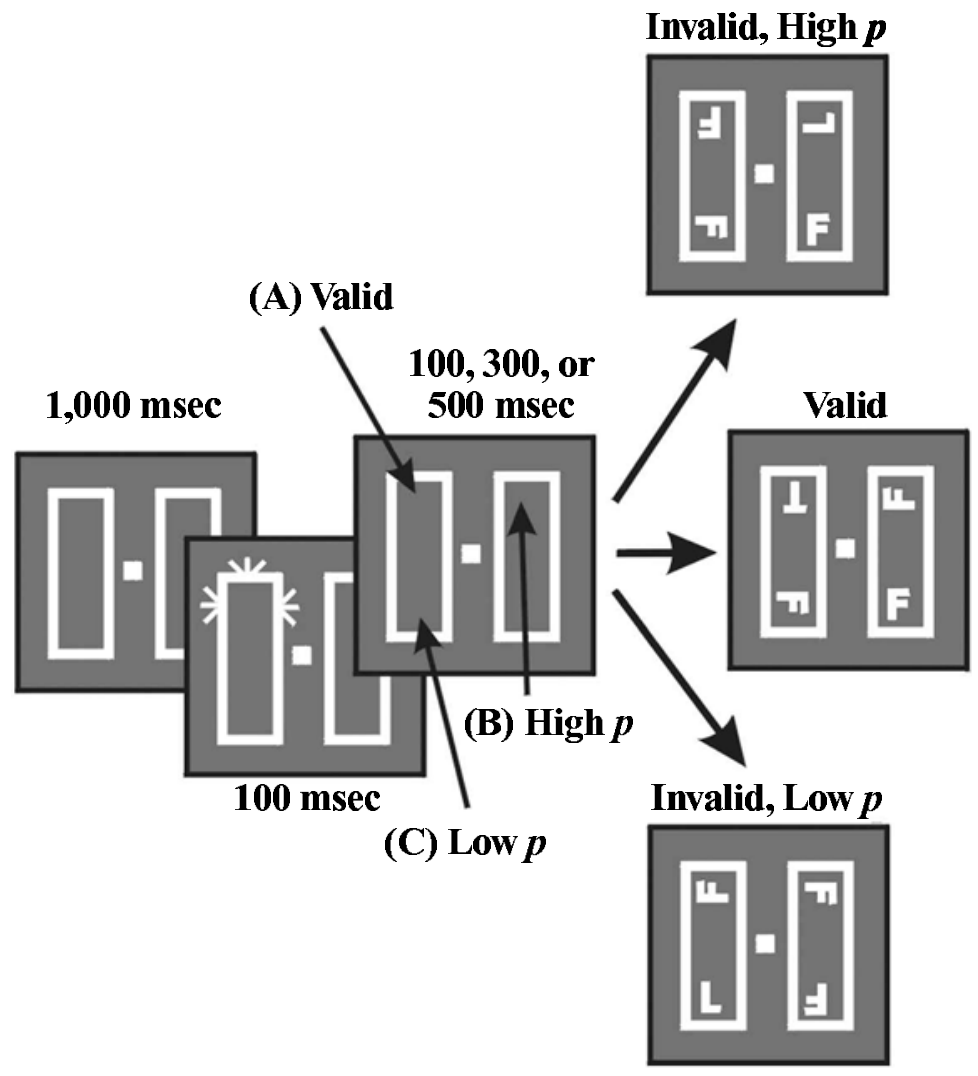

Figure 2. Example display for each of the three conditions of Experiment 2. The task was to perform a $T / L$ letter discrimination among $T / L$ hybrid distractors. (A) Valid target location: The target appears in the cued end of the rectangle. (B) Invalid high-probability location: upper right. (C) Invalid lowprobability location: lower left (this mapping was reversed for half of the subjects). The rectangles were oriented horizontally for half of the subjects; in this case, the high-p/same-object location becomes the high-p/different-object location, and the low-p/different-object location becomes the low-p/same-object location. 
Apparatus and Stimuli. The stimuli were same as in Experiment 1 , with one exception. The four squares were replaced by two rectangles, both oriented either vertically or horizontally (Figure 2 ). Each rectangle subtended $1.2^{\circ} \times 4.2^{\circ}$ with separation of $1.8^{\circ}$ of visual angle between the rectangles. The cue surrounded one end of one of the rectangles.

Design and Procedure. A 3 (validity: valid, invalid same object, invalid different object) $\times 2$ (target location probability: high, low) $\times 3$ (SOA: 200, 400, and $600 \mathrm{msec}$ ) factorial design was used; validity and target location probability were within-subjects factors and SOA was a between-subjects factor. The design was counterbalanced between subjects for rectangle orientation (vertical or horizontal), absolute location of high- and low-probability locations (both pairs of diagonally opposite positions), and the absolute locations of the possible cue events (upper left and lower right for half the subjects; lower left and upper right for the other half). Validity was defined by whether the target appeared in the cued location (valid), at the opposite end of the cued object (invalid same object), or at the end of the uncued rectangle nearest the cue (invalid different object). As in Experiment 1, the cue was valid on $50 \%$ of the trials. The target appeared in the invalid high-probability location on $41.7 \%$ of all trials and in the invalid low-probability location on $8.3 \%$ of all trials. Half of the invalid high-probability trials were sameobject trials and the other half were different-object trials; thus, for $50 \%$ of high-probability trials, regardless of which location was cued, the target appeared within the cued object. Similarly, half of the invalid low-probability trials were same-object trials and the other half were different-object trials.

The procedure was same as in Experiment 1.

\section{Results}

Data from Session 2 only, following the learning session, are reported here. Only the RTs for correct responses were analyzed. RTs greater than $1,500 \mathrm{msec}$ were removed from the analysis, resulting in the discarding of less than $2 \%$ of trials in each SOA condition. Preliminary analysis showed that there were no significant main effects or interactions involving target identity, rectangle orientation, cue locations, or probability locations (all $F_{\mathrm{S}}<1$ ), and therefore the data were collapsed across these conditions. Figure 3 summarizes the findings separately for each SOA.

SOA 200. A one-way repeated measures ANOVA revealed a significant main effect of validity $[F(1,17)=$ 109.44, $p<.001$ ]: RTs for validly cued targets $(M=$ $650 \mathrm{msec})$ were faster than those for invalidly cued targets $(M=713)$, yielding an overall validity effect (i.e., the difference between the valid and invalid conditions) of $63 \mathrm{msec}$. The data from the invalidly cued conditions were then subjected to a 2 (invalid high-probability, invalid low-probability) $\times 2$ (invalid same-object, invalid different-object) repeated measures ANOVA, which revealed that high-probability targets were identified significantly faster than low-probability targets $(M=679$ and $747 \mathrm{msec}$, respectively) $[F(1,17)=101.76, p<$ $.001]$, a probability effect of $68 \mathrm{msec}$. In addition, targets appearing within the cued object were detected faster than those appearing in the uncued object $(M=703$ and $723 \mathrm{msec}$, respectively) $[F(1,17)=13.32, p<.01]$, a 20 -msec object-based advantage. There was no significant interaction between these factors $(F<1)$.

SOA 400. The same ANOVA was applied to the 400msec SOA group, and it revealed significantly faster RTs
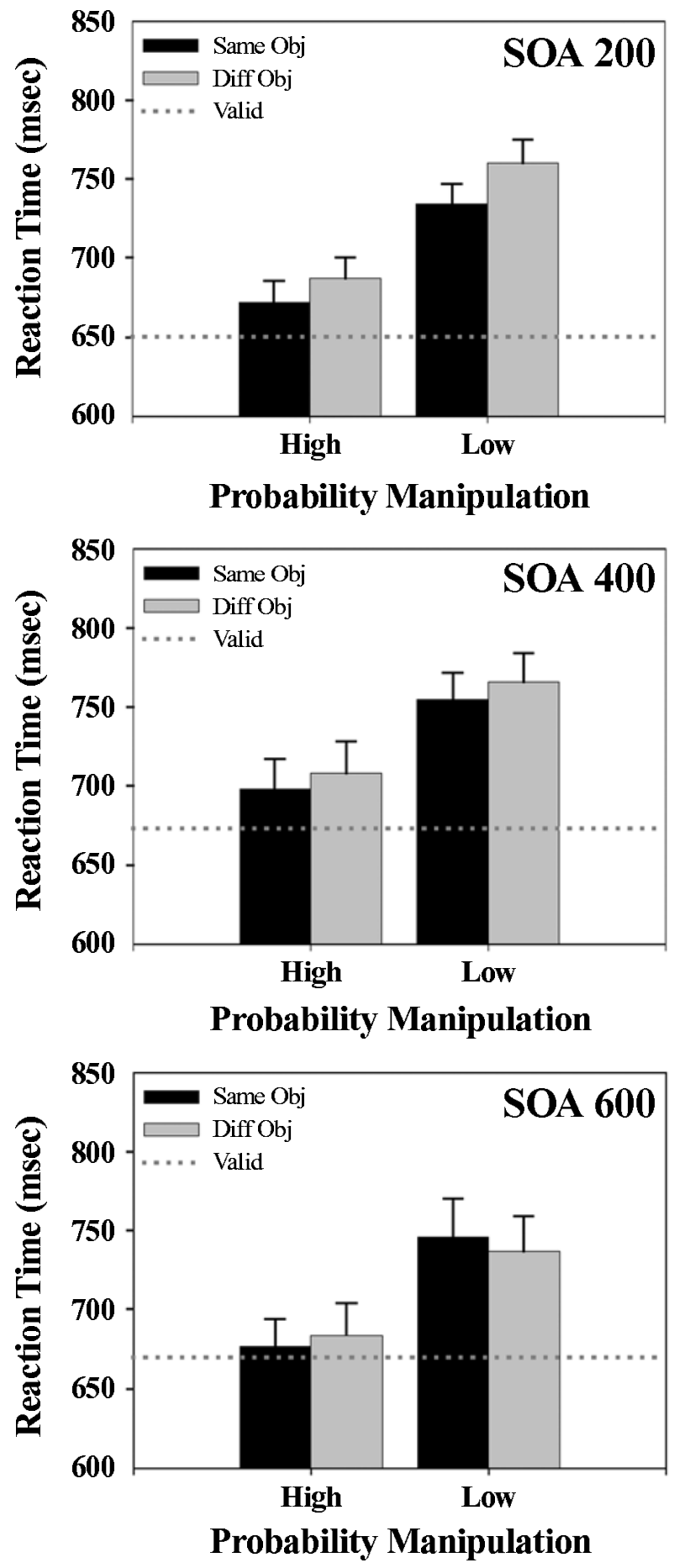

Figure 3. Mean correct reaction times for the invalidly cued trials from Experiment 2 for the three SOA groups. In each panel, the horizontal dashed line represents the valid-cue condition. Error bars represent standard error of the mean.

for validly cued targets $(M=673 \mathrm{msec})$ than for invalidly cued targets $(M=732 \mathrm{msec})$, a 59 -msec validity effect $[F(1,17)=25.26, p<.001]$. For invalidly cued trials, RTs were faster for targets in the high-probability 
location than in the low-probability location $(M=703$ vs. $760 \mathrm{msec}$, respectively), a probability effect of $57 \mathrm{msec}$ $[F(1,17)=37.42, p<.001]$. Targets appearing within the cued object were detected slightly faster than those appearing in the uncued object $(M=726$ and $737 \mathrm{msec}$, respectively), a 10 -msec marginally significant effect $[F(1,17)=2.79, p<.1]$. There was no significant interaction between these factors $(F<1)$.

SOA 600. RTs for valid target identification were significantly faster than those for invalid target types $(M=$ 670 and $711 \mathrm{msec}$, respectively), yielding a $41-\mathrm{msec}$ effect of validity $[F(1,17)=23.92, p<.001]$. Furthermore, an ANOVA on the data from the invalidly cued target condition showed that high-probability targets were identified faster $(M=681 \mathrm{msec})$ than low-probability targets $(M=741 \mathrm{msec})$, a $60-\mathrm{msec}$ probability effect $[F(1,17)=51.52, p<.001]$. No other main effect or interaction was significant (both $F \mathrm{~s}<1$ ).

Finally, an ANOVA was conducted to investigate the interaction between SOA, target location probability, and same versus different object for the invalidly cued trials in two extreme SOAs (i.e., 200 and 600). There was a significant main effect of two factors: probability $[F(1,34)=$ $141.12, p<.001]$ and same versus different object $[F(1,34)=5.04, p<.05]$. More importantly, there was no interaction between SOA and target location probability, suggesting that the magnitude of this effect ( 68 and 60 for the two SOAs, respectively) did not change significantly with SOA $(F<1)$. However, there was a significant interaction between SOA and same versus different object $[F(1,34)=6.21, p<.02]$ : the magnitude of the same versus different object effect declined with SOA [20 msec at the 200-msec SOA and $-2 \mathrm{msec}$ at the 600-msec SOA].

Error rates from each condition are shown in Table 1. Overall, the pattern of error rates paralleled that of the RTs, suggesting the absence of a speed-accuracy tradeoff.

\section{Discussion}

The results of Experiment 2 can be summarized as follows. Subjects in all three SOA groups identified invalidly cued targets more rapidly when they appeared in the high-probability location than when they appeared in the low-probability location, and the magnitude of the probability effect remained relatively constant across the three SOAs $(68,57$, and $60 \mathrm{msec}$ in the 200-, 400-, and 600msec SOA conditions, respectively). The magnitude of the probability effect was also similar to that obtained in

Table 1

Experiment 2: Mean Error Rates for SOAs 200,400 , and 600 Milliseconds

\begin{tabular}{lccccccc}
\hline & \multicolumn{6}{c}{ SOA } \\
\cline { 2 - 4 } \cline { 6 - 8 } & \multicolumn{3}{c}{ High Probability } & & \multicolumn{3}{c}{ Low Probability } \\
\cline { 2 - 4 } \cline { 6 - 8 } & 200 & 400 & 600 & & 200 & 400 & 600 \\
\hline Same object & .01 & .01 & .02 & & .03 & .03 & .05 \\
Different object & .01 & .02 & .02 & & .03 & .03 & .05 \\
\hline
\end{tabular}

Experiment 1 (66 msec). This suggests that the probability manipulation (implicitly learned during the initial session of the experiment) has a consistent and robust effect on attentional prioritization. In contrast, invalidly cued targets appearing within the cued object were identified faster than targets presented in the uncued object- the object-based advantage —only at the shortest SOAs. At the longest SOA, only probability influenced attentional priority. Thus, at the shortest SOAs, we observed the combined effects of contextual and configural factors, whereas at longer SOAs, only contex tual effects contributed to attentional priority.

A concern that might be raised about Experiment 2 is that subjects (particularly in the longer SOA conditions) might have moved their eyes either to the cued location or to a point midway between the cued and the invalid high-probability location (because they had learned that the target would appear in one of those locations about $90 \%$ of the time). Such an outcome would complicate interpretation of the obtained results. The data strongly suggest, however, that subjects did not move their eyes in this way. If they had fixated the cued location, for example, one would have expected a significant decrease in RT for the valid condition with increasing SOA; instead, valid RTs increased slightly. Similarly, if subjects moved their eyes to a point midway between the cued and the high-probability location, then one would expect to observe a relative decrease in RTs for the invalidly cued high-probability location for long versus short SOAs and a relative increase in RTs for the invalidly cued lowprobability location for long versus short SOAs. However, this is not what we observed. The RTs for the highprobability location in SOA 200 and SOA 600 were $M=$ 679 and $M=681$, respectively; the RTs for the lowprobability location in SOA 200 and SOA 600 were $\mathrm{M}=$ 747 and $M=741$ for each SOA, respectively. Together, these data strongly suggest that eye movements did not contribute significantly to the observed pattern of RTs.

\section{GENERAL DISCUSSION}

In the present study, we examined the contribution of configural (object-based) and context-dependent (contingent probability) effects on the deployment of visual attention. Experiment 1 demonstrated that a probability distribution imposed on target locations can be learned and can affect attentional priority. Experiment 2 revealed that when configural and contextual effects were present within the same paradigm at short SOAs, the configural (object-based) and contextual (probability) effects both influenced performance. However, at longer SOAs, the configural contribution disappeared and RTs depended exclusively on the contextual contribution.

This outcome suggests that the frequently observed object-based attentional modulation effect is more complex than is generally believed. We have shown that the object-based effect is a product of at least two types of attentional mechanisms that operate simultaneously. The 
configural mechanism reflects the mandatory object segmentation that accompanies the perception of any complex scene according to well-known principles of perceptual organization (e.g., Palmer, 2002; Palmer \& Rock, 1994). The configural contribution is fast-acting and seems to be a default mode of attentional prioritization. In other words, whenever there is more than one object in the scene, regions within an attended object will, by default, be assigned higher priority for visual exploration than other objects. However, if the behavioral context indicates that some objects or locations should be accorded higher priority than others, then this may dominate the configural bias within a few hundred milliseconds of a cue.

Shomstein and Yantis (2002) demonstrated that objectbased attention is observed only when attention cannot be narrowly focused, suggesting that object-based selection may reflect an object-specific attentional prioritization strategy rather than a mandatory object-based attentional modulation of an early sensory representation. Other researchers have also postulated that object-based modulation could be attributed to some type of strategy. For example, Cepeda and Kramer (1999) observed an inverse object-based effect-that is, a benefit when two target properties appeared on different objects than when they appeared on the same object. The authors suggested that subjects in their experiment used mental rotation and translation in order to perform the task more efficiently, and that this strategy was easier to employ when targets were located on different objects. As in the present Experiment 2, such strategies allow for the suppression of a default object-based attentional allocation schedule and permit a more flexible, context-dependent, distribution of attention.

We suggest that observers are subject to a default configural prioritization schedule (in which unattended locations within an attended object have high priority) that is combined with a more deliberate context-dependent scanning strategy (in which objectively high-probability locations have higher priority), and that their relative influences depend upon task contingencies and the amount of time that is available for the deployment of attention.

\section{REFERENCES}

Avrahami, J. (1999). Objects of attention, objects of perception. Perception \& Psychophysics, 61, 1604-1612.
Behrmann, M., Zemel, R. S., \& Mozer, M. C. (1998). Object-based attention and occlusion: Evidence from normal participants and a computational model. Journal of Experimental Psychology: Human Perception \& Performance, 24, 1011-1036.

Cepeda, N. J., \& Kramer, A. F. (1999). Strategic effects on objectbased attentional selection. Acta Psychologica, 103, 1-19.

Desimone, R., \& Duncan, J. (1995). Neural mechanisms of selective visual attention. Annual Review of Neuroscience, 18, 193-222.

Egeth, H. E., \& YANTIS, S. (1997). Visual attention: Control, representation, and time course. Annual Review of Psychology, 48, 269-297.

EGLY, R., DRIVER, J., \& RAFAL, R. D. (1994). Shifting visual attention between objects and locations: Evidence from normal and parietal lesion subjects. Journal of Experimental Psychology: General, 123, 161-177.

ERIKSEN, B. A., \& ERIKSEN, C. W. (1974). Effects of noise letters upon the identification of a target letter in a nonsearch task. Perception \& Psychophysics, 16, 143-149.

Geng, J. J., \& Behrmann, M. (2002). Probability cuing of target location facilitates visual search implicitly in normal participants and patients with hemispatial neglect. Psychological Science, 13, 520525 .

Miller, J. (1987). Priming is not necessary for selective-attention failures: Semantic effects of unattended, unprimed letters. Perception \& Psychophysics, 41, 419-434.

Moore, C. M., \& EGETH, H. (1998). How does feature-based attention affect visual processing? Journal of Experimental Psychology: Human Perception \& Performance, 24, 1296-1310.

Moore, C. M., Yantis, S., \& Vaughan, B. (1998). Object-based visual selection: Evidence from perceptual completion. Psychological Science, 9, 104-110.

PALMer, S. E. (2002). Perceptual organization in vision. In S. Yantis (Vol. Ed.) \& H. Pashler (Series Ed.), Stevens' Handbook of experimental psychology: Vol. 1. Sensation and perception (3rd ed., pp. 177-234). New York: Wiley.

PALMER, S. E., \& RoCK, I. (1994). Rethinking perceptual organization: The role of uniform connectedness. Psychonomic Bulletin \& Review, 1, 29-55.

Reynolds, J. H., Chelazzi, L., \& Desimone, R. (1999). Competitive mechanisms subserve attention in macaque areas V2 and V4. Journal of Neuroscience, 19, 1736-1753.

Rock, I., \& GUTMAN, D. (1981). The effect of inattention on form perception. Journal of Experimental Psychology: Human Perception \& Performance, 7, 275-285.

SHAw, M. L. (1978). A capacity allocation model for reaction time. Journal of Experimental Psychology: Human Perception \& Performance, 4, 586-598.

Shomstein, S., \& Yantis, S. (2002). Object-based attention: Sensory modulation or priority setting? Perception \& Psychophysics, 64, 4151.

WATSON, S. E., \& KrAmER, A. F. (1999). Object-based visual selective attention and perceptual organization. Perception \& Psychophysics, 61, 31-49.

(Manuscript received September 26, 2002;) revision accepted for publication February 12, 2003.) 